\title{
Erratum to: Dzhuraev's Formulas and Poly-Bergman Kernels on Domains Möbius Equivalent to a Disk
}

\author{
Luís V. Pessoa
}

Published online: 2 October 2014

(C) Springer Basel 2014

\section{Erratum to: Complex Anal Oper Theory (2013) 7:193-220 DOI 10.1007/s11785-010-0119-9}

We proceed with the necessary modifications in the hypotheses of the auxiliary result Proposition 3.5 of the originally published article.

Theorem 1.1 Let $\mathcal{H}_{k}(k=1,2,3)$ be Hilbert spaces and let $P: \mathcal{H}_{1} \rightarrow \mathcal{H}_{2}$ and $V: \mathcal{H}_{2} \rightarrow \mathcal{H}_{3}$ be partial isometries with initial spaces $N_{1}$ and $N_{2}$ and final spaces $M_{1}$ and $M_{2}$, respectively. Then $V P$ is a partial isometry if and only if the projections $V^{*} V$ and $P P^{*}$ commute. If $V P$ is a partial isometry, then its initial and final spaces are $P^{*}\left(N_{2} \cap M_{1}\right)$ and $V\left(N_{2} \cap M_{1}\right)$, respectively.

Theorem 1.1 corrects Proposition 3.5 of the original article. In the original article the mentioned proposition works as an auxiliary result for the proofs of some of the main results of the paper. It is used there to compute the initial and final spaces of the following partial isometries (see above Proposition 3.6 of the original article and Corollary 3.9)

$$
T_{n}:=\chi_{\Omega} S^{*} \chi_{\mathbb{D}} S_{n} \chi_{\Omega} \quad \text { and }\left(S_{\Omega}^{*}\right)^{k} T_{n} \quad(n, k=1, \ldots) .
$$

The online version of the original article can be found under doi:10.1007/s11785-010-0119-9.

This work was partially supported by FCT project PEst-OE/MAT/UI4032/2011.

L. V. Pessoa $(\varangle)$

Departamento de Matemática, Instituto Superior Técnico,

Av. Rovisco Pais, 1049-001 Lisboa, Portugal

e-mail: 1pessoa@math.ist.utl.pt 
For the reader convenience, in the following result we explain how to justify the validity of the hypothesis missing in Proposition 3.5 of the original article, for each use of the later Proposition. To begin with, we introduce the following definition

$$
T_{j, n}:=\chi_{\Omega} S_{j} \chi_{\mathbb{D}} S_{n} \chi_{\Omega} \quad\left(j, n \in \mathbb{Z}_{ \pm}\right)
$$

For $n=1,2, \ldots$, we note that $T_{n}=T_{1, n}$.

Theorem 1.2 Let $k$ and $m$ be positive integers and let $j$ and $n$ be non-zero integers. Then $T_{j, n}$ and $\left(S_{\Omega}^{*}\right)^{k} T_{m, n}$ are partial isometries on $L^{2}(\Omega)$.

Proof Define the following operator

$$
\mathcal{V}_{j}: L^{2}(\mathbb{D}) \rightarrow L^{2}(\Omega), \quad \mathcal{V}_{j}:=\chi_{\Omega} S_{j} \chi_{\mathbb{D}}
$$

We known that a bounded operator $P$ between Hilbert spaces is a partial isometry if and only if $P^{*} P$ is a projection. Thus, that $\mathcal{V}_{j}$ is a partial isometry follows from the Dzhuraev's formulas over the unit disk $\mathbb{D}$, which are equivalent to $B_{\mathbb{D}, j}=\mathcal{V}_{j}^{*} \mathcal{V}_{j}$ (see also Proposition 3.3 of the original article). Moreover, $T_{j, n}$ coincides with the product of the partial isometries $\mathcal{V}_{j} \mathcal{V}_{-n}^{*}$. Since $B_{\mathbb{D}, j}=\mathcal{V}_{j}^{*} \mathcal{V}_{j}$ and $B_{\mathbb{D},-n}=\mathcal{V}_{-n}^{*} \mathcal{V}_{-n}$ and we know that the projections $B_{\mathbb{D}, j}$ and $B_{\mathbb{D},-n}$ commute (see [1, Lemma 3.1]) then from Theorem 1.1 it follows that $T_{j, n}$ is a partial isometry. Finally we show that $\left(S_{\Omega}^{*}\right)^{k} T_{m, n}$ is a partial isometry. Since $T_{m, n}$ is a partial isometry, then $T_{m, n} T_{m, n}^{*}$ is a projection whose image is contained in the final space of $\chi_{\Omega} S_{m} \chi \mathbb{D}$, which by Theorem 2.8 of the original article we know that is a subspace of $\mathcal{A}_{-m}^{2}(\Omega)$. Moreover, $\left(S_{\Omega}\right)^{k}\left(S_{\Omega}^{*}\right)^{k}$ is the projection onto the orthogonal space of $\mathcal{D}_{k}(\Omega)$. Since $\mathcal{D}_{k}(\Omega) \subset \mathcal{A}_{k}^{2}(\Omega)$ (see Corollary 3.4 of the original article) and $B_{\Omega, k} B_{\Omega,-m}=0$ (see Corollary 2.6 of the original article) then the final space of $T_{m, n}$ is contained in the initial space of $\left(S_{\Omega}^{*}\right)^{k}$. From Theorem 1.1 we then obtain that $\left(S_{\Omega}^{*}\right)^{k} T_{m, n}$ is a partial isometry.

\section{Reference}

1. Karlovich, Yu.I., Pessoa, L.V.: Poly-Bergman projections and orthogonal decompositions of $L^{2}$-spaces over bounded domains. Oper. Theory Adv. Appl. 181, 263-282 (2008) 